\title{
ABORDAJES DE COMPRENSIÓN INTERDISCIPLINARIOS. DIÁLOGO ENTRE EL DERECHO Y LA TEORÍA DEL DISCURSO, A PARTIR DE UN ESTUDIO DE CASO: VILLA LA TELA EN LA CIUDAD DE CÓRDOBA.
}

\section{COLLABORATIVE APPROACHES TO UNDERSTANDING. DIALOGUE BETWEEN THE LAW AND THE THEORY OF DISCOURSE, FROM A CASE STUDY: VILLA LA TELA IN THE CITY OF CÓRDOBA}

\section{AUTORES}

Mariana Jesús Ortecho: Becaria doctoral del Consejo Nacional de Investigaciones Científicas y técnicas (Conicet). Sede en Centro de Estudios Avanzados (CEA). Córdoba (Argentina). mensajedeletras@hotmail.com

María Florencia Pasquale: Becaria doctoral del Consejo Nacional de Investigaciones Científicas y técnicas (Conicet). Sede en Centro Experimental de la Vivienda Económica (Ceve). Córdoba (Argentina). fpasquale@ceve.org.ar

\section{RESUMEN}

Enmarcado en un proyecto de investigación-acción, el presente trabajo recupera un conjunto de reflexiones en torno a los aportes que las Ciencias Sociales, especialmente el Derecho y la Teoría del Discurso, pueden realizar a procesos de conflicto inter-sectorial urbano. A través de un caso específico, el asentamiento de Villa La Tela en la ciudad de Córdoba-Argentina, se revisan una serie de conceptualizaciones teóricas provenientes de las áreas de conocimiento antes mencionadas, a la luz de las demandas comunicacionales que este conflicto en particular y este tipo de situación en general 
exige. El conjunto de la propuesta intenta asumir la complejidad de la problemática desde una perspectiva analítica interdisciplinar operacionable en procesos empíricos.

\section{PALABRAS CLAVE}

Investigación-Acción - Interdisciplina - Derecho - Teoría del Discurso - Relaciones Intersectoriales.

\section{ABSTRACT}

From an action research project, this paper brings a set of reflections on the contributions that Social Sciences, especially the Law and the Theory of Discourse, can make to the processes of inter-sectoral urban conflicts. Through an specific case, the settlement of Villa La Tela in the city of Cordoba-Argentina, a series of theoretical conceptualizations are reviewed from the fields of knowledge mentioned above. Considering the communication demands that this particular conflict and this type of situation usually requires, the entire proposal seeks to take on the complexity of the problem. Therefore the analytical and interdisciplinary perspective attempts to operationalize its concepts into empirical tools.

\section{KEY WORDS}

Investigation-Action - Interdisciplinary- Law - Theory of Discourse - Intersectorial Relations.

\section{ÍNDICE}

1. INTRODUCCIÓN

2. ASENTAMIENTO VILLA LA TELA, UN CASO PARADIGMÁTICO

2.1 Desarrollo urbano latinoamericano 
2.2 Villa La Tela: el conflicto jurídico sobre el suelo y la dimensión interactoral

3. APORTES DESDE LA INTERDISCIPLINA:

3.1 Las intervenciones públicas y el modelo de Estado

3.2 La perspectiva jurídica:

3.2.1 El cambio de paradigma jurídico

त 3.2.2 El derecho como discurso

7 2.3 La mediación como herramienta discursiva

73.2.4 Las características de la mediación y sus herramientas

3.3 Los Estudios sobre Discurso en el marco de la indagación científica cualitativa.

14. A MODO DE CONCLUSIÓN

5. BIBLIOGRAFÍA

\section{INTRODUCCION}

El presente trabajo forma parte de un proyecto de investigación aprobado por el Ministerio de Ciencia y Tecnología de la provincia de Córdoba-Argentina, con sede en el Centro de Estudios Avanzados - CEA, y el Centro Experimental de la Vivienda Económica - CEVE. Las autoras forman parte del equipo interdisciplinario que desde fines de 2009 a la fecha, se encuentra trabajando con la comunidad de Villa La Tela, asentamiento ubicado en la misma ciudad. A partir de sucesivas instancias de encuentro con los vecinos, y del enriquecimiento que estas experiencias representan, este trabajo aborda cuestionamientos sobre las implicancias del diálogo inter-actoral.

Este texto pretende reflexionar sobre los aportes que las Ciencias Sociales, especialmente el Derecho y la Teoría del Discurso, pueden realizar al momento de abordar relaciones inter-sectoriales atravesadas por tensiones propias del modelo de desarrollo de las ciudades occidentales. La propuesta es tomar elementos de un estudio 
de caso: Villa La Tela en la ciudad de Córdoba, para reflexionar sobre las contribuciones que las mencionadas disciplinas pueden realizar al abordar determinados conflictos urbanos en escenarios sociales complejos.

La línea argumental del trabajo se inicia con una presentación de la situación urbana latinoamericana como marco en el que se contextualiza a la comunidad de Villa La Tela. Posteriormente se presenta a los distintos sectores afectados y al conflicto jurídico sobre el suelo, en tanto componentes constituyentes entre los cuales se desenvuelven las relaciones inter-actorales.

Los aportes que se pretenden realizar desde la interdisciplina se estructuran en las dos ramas mencionadas: el Derecho y la Teoría del Discurso. La perspectiva jurídica realiza un planteo a partir de la alternativa al paradigma judicial-contencioso que representa la institución de la mediación. A su vez los Estudios sobre Discurso, como parte de la indagación científica cualitativa, se presentan como dispositivo conceptual capaz de comprender los procesos de construcción social de sentido que establecen las tensiones que configuran el conflicto en cuestión.

\section{ASENTAMIENTO VILLA LA TELA, UN CASO PARADIGMÁTICO.}

\subsection{DESARROLLO URBANO LATINOAMERICANO.}

Muchas de las metrópolis latinoamericanas, pero también de otras regiones del mundo occidental u 'occidentalizado', presentan una serie de características similares que aún sin aparecer como idénticas, señalan un patrón social recurrente en el modo de construir ciudad.

La relación centro-periferia es una pauta vincular que se repite, tanto en la dimensión física como simbólica de las grandes urbes. 
Toda ciudad presenta espacios diferenciados, zonas que con mayor o menor prolijidad se corresponden con la estratificación socio-económica de la población, generando ámbitos muy diversos en términos culturales e identitarios.

Las redes simbólicas que se generan sobre estas sub-divisiones del espacio físico citadino van erigiéndose entre los propios habitantes de una ciudad de un modo invisible pero rotundo, generando juicios y múltiples prejuicios entre los diferentes grupos humanos que animan estos escenarios.

Estas tensiones entre sectores son generadoras de múltiples situaciones de confrontación social. La fragmentación del tejido urbano deviene generalmente en diversos conflictos que deben ser leídos en su complejidad al intentar comprender la realidad de las ciudades latinoamericanas.

Estas características, propias del modelo de desarrollo de las ciudades occidentales, cuyo origen resida probablemente en las relaciones de producción y distribución de recursos materiales y culturales, se presenta en cada situación de un modo particular. Por ello, la comprensión específica de cada uno de estos procesos es necesaria y útil, aunque de ninguna manera pueda ser empleada como descripción genérica. Sin embargo, un caso específico puede observarse y considerarse paradigmático, en tanto ciertos aspectos constituyan buen ejemplo de otras situaciones similares que integren un conjunto mayor o universo general.

Numerosas ciudades de América Latina han desarrollado, fundamentalmente en la segunda mitad del siglo veinte, un importante número de asentamientos humanos espontáneos que, desde su constitución, escapan a los cánones de formalidad que el Estado reconoce y exige.

El fenómeno de emergencia y reproducción de estos espacios, impulsado fuertemente por los procesos de industrialización de las economías nacionales y las consecuentes 
migraciones provenientes de los ámbitos rurales, puede leerse como producto de la deficiente absorción laboral que las ciudades 'prometieron' a los sectores antes ligados a la producción agraria.

La ocupación de tierras, organizada o gradual y desarticulada, por parte de las comunidades marginadas del sistema económico y cultural es un episodio recurrente y, en rigor, característico del rumbo de crecimiento de las urbes latinoamericanas.

\subsection{VILLA LA TELA: EL CONFLICTO JURÍDICO SOBRE EL SUELO Y LA DIMENSIÓN INTER-ACTORAL.}

Villa La Tela es un asentamiento espontáneo que aparece aproximadamente en 1970 en uno de los núcleos urbanos más grandes de Argentina, la ciudad de Córdoba. Conformada hoy por más de 500 familias, esta comunidad se ha constituido a lo largo de cuatro décadas en un grupo social que se describe frecuentemente a sí mismo como heterogéneo. Sin embargo, sus habitantes perciben y asumen explícitamente una pertenencia identitaria que, aunque transitoria, está determinada por la ocupación de ese específico espacio en la ciudad.

Como población atravesada por los efectos de diferentes programas asistenciales diseñados desde un marco de políticas sectoriales, esta comunidad encuentra un modo de subsistencia diario fuertemente sujeto a la actividad de comedores infantiles y a planes de asignación familiar.

Por otra parte, Villa La Tela presenta una situación compleja en términos organizacionales ya que por una parte cuenta con escasas y muy enfrentadas organizaciones sociales endógenas, pero siendo a su vez intensamente intervenida por instituciones externas, tales como organizaciones no gubernamentales, grupos religiosos y distintos grupos académicos. 
Aún constituyendo una situación única que debe ser comprendida en su singularidad, este asentamiento puede ser tomado como caso paradigmático o ejemplo expresivo para conocer y analizar la forma en que este tipo de comunidad se ubica en el entramado de vinculaciones inter-sectoriales urbanas.

Así por ejemplo, resulta relevante destacar que la mayoría de los grupos familiares que fueron, en distintos momentos, estableciéndose en este espacio no goza de seguridad jurídica sobre sus viviendas. Es decir que en primer término y al momento mismo de radicación, estas familias iniciaron, aunque indirectamente, una relación virtual con los propietarios legales de estos terrenos.

Por otra parte, y debido a la ubicación en el entramado urbano, uno de los actores que muy posiblemente emerja a futuro en este contexto, es el del inversionista privado con intereses en el usufructo comercial de estas tierras, que desde luego son un importante, aunque aún potencial, capital inmobiliario.

El conflicto latente que este asentamiento plantea es, por supuesto, el de la disputa por la tierra. La denuncia por usurpación de terrenos que culmine en el desalojo forzado de estas familias es una amenaza considerable.

El Estado, por su lado, tiene asimismo incidencia directa en este asunto mediante sus diversas instancias: municipal, provincial y nacional, como instituciones reguladoras desde los ámbitos ejecutivos, legislativos y por supuesto judiciales.

Por último observamos que, de modo tangencial pero no menos importante, aparece el rol de los vecinos residentes de la zona en cuestión. En este caso, y frecuentemente en otros similares, las poblaciones contiguas suelen resistir fuertemente a la permanencia de estos grupos dentro del ejido urbano. Sucede que la precarización material de las condiciones de vida trae aparejada la delincuencia como un efecto que desborda e 
impacta más intensamente en los espacios colindantes contribuyendo a la intolerancia cultural que generalmente distancia a grupos de diferente estrato social.

Por lo mencionado anteriormente, el suelo urbano aparece, de este modo y en este caso, como un objeto de interés en torno al cual se ubican una serie de actores: actuales ocupantes, titulares dominiales, entidades de gobierno y vecinos de barrios adyacentes. Pero esta situación desde luego viene asociada a otros asuntos de orden social general que provocan la participación de muchas agrupaciones que se acercan, desde diferentes temáticas, a realizar investigaciones y acciones orientadas al Desarrollo Social. Este es el caso, por ejemplo, de centros de investigación que dan lugar a proyectos tales como el que suscita la escritura del presente artículo.

\section{APORTES DESDE LA INTERDISCIPLINA:}

Las relaciones esbozadas son, como se dijo, vinculaciones efectivas con espesor histórico en algunos casos y sólo potenciales en otros. Lo que resulta importante aquí, es poner en consideración la complejidad del entramado que este tipo de situación representa en términos de diálogo inter.-actoral.

Cada uno de los lazos establecidos constituye un desafío, una posibilidad de fricción y conflicto, como también una oportunidad de crecimiento ciudadano.

La labor de encontrar las categorías analíticas y los marcos conceptuales pertinentes para comprender la realidad socio-económico-jurídica del caso planteado es una tarea constante a desempeñar en el curso de la investigación.

Tal como lo expusimos anteriormente, el eje de la indagación parte del reconocimiento de la complejidad del entramado de relaciones interactorales, poniendo énfasis en la disputa jurídica por la tierra. 
Para esto abordaremos el presente trabajo partiendo del modelo de Estado vigente, para luego realizar aproximaciones desde el Derecho, la Metodología Cualitativa y el Análisis del Discurso.

\subsection{LAS INTERVENCIONES PÚBLICAS Y EL MODELO DE ESTADO.}

El abordaje de problemas sociales, como los señalados, ha permitido la aplicación de políticas de resolución intervencionista; siendo los procesos de ocupación territorial casos característicos de esta estrategia de acción. La relocalización de asentamientos espontáneos en espacios que están por fuera de la ciudad, en términos geográficos y simbólicos, ha constituido un claro ejemplo de un tipo de gestión gubernamental provincial que sufrió la propia ciudad de Córdoba.

Ahora bien, es importante considerar que la posibilidad de concretización de estas disposiciones ejecutivas tiene lugar en el marco de un modelo de relación entre Estado y Sociedad Civil, que quizás se remonte al proyecto de la figura estatal propuesto por la modernidad europea.

La obligación y el derecho, que aparecen como verso y reverso de una misma vinculación, serían el cordón más fuerte que al menos se supuso, debió sostener la estructuración social de la civilización occidental en los últimos siglos.

Aquella entidad supra-individual, habitada por diferentes actores gubernamentales, encuentra en la ley el precepto o la palabra normativa que determina dogmáticamente el límite entre lo aceptable y lo condenable en las conductas humanas. Sin embargo, es lógico que debido a las diferentes posiciones que un individuo o un grupo adquiera, este precepto aparezca frecuentemente como postulado ambiguo, operando en algunas situaciones como amparo y en otras como coerción. 
La imposición legal vertical, desde el Estado al sector Civil, debería consistir en una instancia lateral en una sociedad que aspire al desenvolvimiento de sus propias capacidades y aptitudes, reservando sus esfuerzos a fortalecer las relaciones y acuerdos horizontales entre sus miembros. La diferencia reside probablemente, en el propósito profundo y último. En el primer caso, la acción 'intervencionista' privilegia la búsqueda de resultados en términos de disolución de conflictos o control social. De modo diferente, aunque no contrario, la apuesta por la calidad en los procesos de diálogo y comunicación entre diversos sectores aspira a generar procesos de aprendizaje, entendiéndolos también como parte de los resultados deseados.

Por ello, el interés por verter parte de los conocimientos desarrollados desde un área del saber como es la Teoría del Discurso a una situación de conflicto inter-actoral reside no sólo en el propósito de contribuir a la resolución de estas situaciones, sino que de modo profundo se reconoce como una práctica tendiente a la transformación de las relaciones estructurales que sostienen el andamiaje social.

En el mismo sentido se rescatan aquí los aportes de la práctica de mediación en tanto institución que, partiendo de la valorización del protagonismo de los sujetos, representa una alternativa a la resolución de conflictos que se va abriendo camino en distintos ámbitos sociales ${ }^{1}$.

Al rescatar los aportes teóricos mencionados e intentar estrechar vínculos con las prácticas en que los mismos convergen, se considera que la promoción de la comunicación inter-actoral calificada, al proponer cambios en las aptitudes civiles, trae de algún modo aparejada la idea de transformación de la figura del propio Estado. En este sentido, podría asociarse esta manera de comprender las articulaciones sectoriales fundantes de 'lo social' a lo señalado por Boaventura de Sousa Santos (2005:91) y a

1 Si bien el ámbito más conocido es el "judicial”, se aplican las herramientas a prácticas comunitarias, esto es problemas vecinales o escolares, entre otros. 
propósito de las nuevas funciones del sector estatal y civil. Las obligaciones políticas actuales, señala el autor, no debieran quedar restringidas a la verticalidad de la ligadura entre ciudadanos y Estado, sino que debieran extenderse y fortificarse en las vinculaciones horizontales que suscitan otro concepto muy diferenciado: el de comunidad.

\subsection{LA PERSPECTIVA JURÍDICA:}

\subsubsection{EL CAMBIO DE PARADIGMA JURÍDICO.}

El positivismo dogmático representa el paradigma iusfilosófico aún imperante en la concepción de numerosos operadores jurídicos. Estudios referidos a la formación universitaria en las facultades de derecho, validan esta afirmación ${ }^{2}$. Hay una reducción en la comprensión del fenómeno jurídico que lleva a un distanciamiento de la realidad empírica.

El reconocimiento de una realidad jurídica en "sentido amplio" obliga, necesariamente, a acudir a enfoques que permiten incorporar perspectivas de análisis, aportando nuevos elementos a la comprensión de la realidad y, correlativamente, contribuyen a la formulación de enfoque superadores.

Cárcova en su obra "Teorías jurídicas postpositivistas" (2009:15) se refiere al malestar de la teoría a partir de la crisis de los presupuestos cartesiano-positivistas afirmando que el punto de partida estaría en que el saber que se ocupa de la interacción social debe tener como finalidad más que explicar, comprender. De este modo, y desde la propuesta fundante de Dilthey que sugería emplear la empatía como recurso para la construcción del conocimiento social, la reflexión jurídica actual puede beneficiarse del

2 Pueden consultarse trabajos de Carlos Lista (2001) sobre la formación universitaria d la Facultad de Derecho de la Universidad Nacional de Córdoba: “La construcción de la conciencia jurídica: Los Objetivos Educativos y la formación del abogado". Anuario V, Centro de Investigaciones Jurídicas y Sociales, Facultad de Derecho y Ciencias Sociales, U.N.C., v.1, n.1, p.381 - 404. 
diálogo y recuperación de ciertas elaboraciones provenientes de diversas corrientes como el postestructuralismo o la teoría de sistemas, por ejemplo, que aporten elementos para complejizar la reflexión en torno a la práctica del derecho.

\subsubsection{EL DERECHO COMO DISCURSO}

Francois Ost (1992) presenta una ilustrativa metáfora referida al modelo de juez y de derecho que representan las alternativas de cómo interpretar el rol social de este último. El primero, el juez "Júpiter", sería aquel que desde arriba señala en forma imperativa fundándose en la ley; el segundo, el juez de Dworkin que él llama juez “Hércules”, sería aquel semidiós que carga al mundo sobre sus brazos y que es capaz de desenvolver todas las tareas. Frente a estos dos modelos, Ost propone ver al derecho como "Hermes", el mensajero de los dioses. El que preside la comunicación y la circulación. Así como la pirámide y el embudo simbolizan a los dos primeros, la imagen de una red representaría a la última metáfora según el autor.

El derecho aparece así como discurso, como circulación constante de sentido. Aquí, si bien se distinguen los operadores, no hay prevalencia de unos sobre otros, será derecho tanto lo que diga la ley, como lo que interpreten los técnicos del derecho, esto es jueces y abogados, doctrinarios, así como también los ciudadanos sometidos al imperio de las normas. Profundizando el análisis desde esta perspectiva, podría decirse que existen constelaciones de sentido jurídico que se pueden dar por fuera del derecho legislado e institucionalizado, pero que estarían formando parte de un derecho en sentido amplio.

Boaventura de Sousa Santos (2002) también reflexiona sobre la teoría dialógica, y la coloca en un lugar central de su propuesta de salida a la crisis del paradigma dominante en que, asume, se encuentra el derecho y la ciencia en la actualidad. Su teoría de la traducción avanza en este sentido. Él afirma que "una justicia social global sólo se dará con una justicia cognitiva global"(2002:240). El autor parte de una 
hermenéutica diatopical, esto es, la idea de que todas las culturas son incompletas y pueden ser enriquecidas del diálogo con otras culturas.

La traducción es el "procedimiento que permite una inteligibilidad mutua entre las diversas experiencias del mundo, con el objetivo de crear constelaciones de saberes y prácticas lo suficientemente fuertes como para representar alternativas creíbles a lo que llamamos globalización neoliberal (2002:274)". Esto implica reconocer y dialogar con experiencias hasta ahora dejadas de lado por la lógica reguladora del capitalismo postmoderno.

En el campo jurídico implicaría abrir el concepto de lo que entendemos por derecho a otras experiencias, que si bien tienen existencia sociológica, en cuanto existen supra o infra estatalmente, no son reconocidas por el derecho estatal. Y este es precisamente el centro de la propuesta: pensar al derecho separado del Estado, no para dejar de lado el derecho legal, pero sí para considerar su coexistencia e interrelación real con otras prácticas sociales que también deberían calificarse como jurídicas.

\subsubsection{LA MEDIACIÓN COMO HERRAMIENTA DISCURSIVA.}

La institucionalización y la correlativa práctica de resolución de conflictos estuvo reservada tradicionalmente al proceso judicial. Sin embargo, el intento por superar el paradigma positivista ha repercutido en la manera de mirar las respuestas que el derecho ofrece a las problemáticas sociales. En este sentido en el apartado anterior intentamos plantear una de las miradas post-positivistas que aporta la sociología jurídica crítica al proponer la inclusión de los sectores regulados por el derecho como sectores activos en la creación del mismo.

Desde esta postura nos interesa abordar las características de la mediación en tanto institución que se presenta como alternativa a la hora de resolver conflictos intersectoriales comunitarios. 
Al igual que otros países, Argentina ha desarrollado en los últimos años métodos de Resolución Pacífica de Conflictos. La mediación, como uno de estos métodos, se presenta como una herramienta con potencialidad de abordar el conflicto con habilidades que flexibilizan el manejo técnico-jurídico en la composición de intereses.

Tomar conciencia de la trama compleja de las relaciones interactorales, los intereses que motivan las respectivas posiciones, y las significaciones construidas por cada grupo, implica afrontar el desafío de exigirle una sensibilidad distinta a las instituciones tradicionales encargadas de resolver controversias. Implica asumir que debe mediar una lógica distinta al abordar la resolución del conflicto.

En este sentido, interesan los aportes que la mediación puede hacer favoreciendo el diálogo ante conflictos intersectoriales urbanos. La mediación comunitaria se constituye una instancia que puede iluminar el complejo proceso de búsqueda de consenso. Entendiendo éste no como unanimidad sino como proceso de compromiso y convergencia en continuo cambio entre convicciones divergentes (Sartori:2001).

En el ámbito comunitario, la mediación pretende ubicar a los sujetos en roles protagónicos. Se sustituye así la atribución de responsabilidades impuesta por el sistema judicial, por la composición de intereses en un ámbito de diálogo intersectorial con acompañamiento profesional (mediador especializado).

De esta manera, se presenta la posibilidad de implementar herramientas de mediación ante las asimetrías planteadas en la relaciones de los vecinos de Villa La Tela con los vecinos de los barrios cercanos; frente a las diferencias que los mismos pueden tener con un posible inversor inmobiliario; o frente al municipio en tanto sujeto público obligado a proveer ciertas condiciones mínimas de habitabilidad, salud, educación, etc, para con los habitantes, en este caso, de un sector socio-económico vulnerable. 


\subsubsection{LAS CARACTERÍSTICAS DE LA MEDIACIÓN Y SUS HERRAMIENTAS}

Uno de los rasgos que define la institución de la mediación es la inclusión de una tercera figura ajena al conflicto, el mediador, que ayuda a las partes a llegar a un acuerdo, pero cuyas intervenciones no tienen carácter vinculante, a diferencia de la sentencia judicial.

A lo largo de las tres últimas décadas se han desarrollado, y por tanto hoy pueden identificarse, tres modelos de mediación. Marines Suares (2005) expresa que en un extremo estaría el Modelo Tradicional, que deriva del campo de los negocios, centrado fundamentalmente en el acuerdo. En el otro extremo se encontraría el modelo transformativo, más atento a la dimensión relacional, y por tanto más próximo a la Psicología. Finalmente y, entre estos dos, la autora ubica al Modelo Circular-Narrativo de Sara Cobb, orientado tanto a la modificación del aspecto vincular como a la obtención de un acuerdo. Este último, al que adherimos a los fines de este trabajo, tiene vinculación con la línea de terapia sistémica y con las teorías postestructurales de las narrativas.

La relación con la producción de lo que fue el Mental Research Institute (MRI), influenciado fuertemente en sus comienzos por Gregory Bateson, puede entenderse como una necesidad de incluir herramientas que permitieran comprender a los grupos protagonistas de los conflictos -familias en primer término, pero luego cualquier ámbito intersubjetivo - como un sistema de interrelaciones.

Por otra parte, es importante destacar que este modo de 'intervención familiar' fue fortaleciéndose fuera de los propios Estados Unidos generando, por ejemplo, el surgimiento de numerosas escuelas de terapia familiar sistémica en Europa ${ }^{3}$.

3 Marines Suares menciona entre estas a la Escuela de Milán, dirigida por Mará Selvini Palazzoli, quien se había formado en el MRI de Palo Alto, California; también se han destacado la Escuela de Roma, de Maurizio 
La idea central de esta propuesta radica en considerar a todo grupo como un sistema que al desarrollar un rasgo disfuncional puede corregirse con la inclusión de un nuevo elemento. "No es necesario cambiar a la totalidad del sistema; la introducción de un cambio, por pequeño que sea, si es mantenido, produce efectos en la totalidad del sistema (Sic)"(Marines Suárez 2005:167). De esta manera, las terapias breves se focalizan en aspectos particulares, intentando lograr pequeños cambios, los cuales, por tener conexiones con la red general, se extenderán hacia otros elementos o relaciones de éstos.

Las técnicas que propone el modelo circular narrativo parten de asumir que las personas que vivencian un conflicto han construido relatos o historias constituidas por argumentos que funcionan como descripciones o interpretaciones de hechos. Estas narraciones se establecen y naturalizan de forma tal, que llevan a las personas a percibir y comprender desde "marcos estrechos" que impiden mirar el conflicto más allá de la construcción subjetiva que se ha realizado de la situación. La autora citada en el párrafo anterior explica que se trata de ayudar a las personas a "desempantanarse", esto es, desestabilizar los relatos que las partes traen para dar lugar a que pueda surgir una historia alternativa y, posiblemente, una correlativa relación distinta.

Entre las técnicas que favorecen la búsqueda de protagonismo de las partes, se privilegian las preguntas que tengan la característica de ser lo "suficientemente inusuales" como para producir cambios, al decir de Tom Andersen (1994). El modo interrogativo tiene la virtud de dar lugar a la participación de los sujetos que podría llevar a modificar la relación que siempre han mantenido, y la manera como siempre han resuelto sus diferencias. 
Para lograr este fin se pueden utilizar diversos instrumentos. Una herramienta privilegiada es la Pregunta Circular. Dicha intervención pretende lograr que las partes comprendan la interdependencia entre los elementos de un sistema, de manera tal que sean conscientes de su relativa supeditación respecto del conjunto dentro del cual se deben comprender los deseos y las posibilidades. El interesante efecto que puede darse, si estos elementos son comprendidos, permite que los sujetos comiencen a actuar en consecuencia, esto es, que consideren que es inevitable e importante llegar a ciertos entendimientos como condición mínima de la vida en comunidad.

El hecho de reflexionar sobre la manera de pensar de otra persona crea un circuito de interacción que posibilita comprender la relación en términos de causalidad circular.

En definitiva lo que se persigue con este tipo de intervenciones es una transformación en la manera de comprender y representar las situaciones de tensión y conflicto. Este cambio se produciría cuando se da una modificación en el sujeto, es decir, cuando se crea una diferencia debido a que se reestructuró la manera de pensar y por tanto reaccionar frente a determinadas situaciones. Este mecanismo, que la teoría cibernética de segundo orden llama feedback positivo, consiste en provocar la ruptura de ciertas secuencias de acciones, como medio para suscitar nuevas emociones, y por tanto, futuras y diferentes reacciones.

Por otra parte, este mismo proceso constituye aquello que Bateson llamó "deuteroaprendizaje". En este caso se trata de recuperar el mecanismo que se utilizó al solucionar un conflicto para abordar futuras situaciones. Esto ha recibido también el nombre de "transferencia de aprendizaje" o "conocimiento tácito". Suares (2005) afirma que a veces las partes no son conscientes de este aprendizaje en el momento en que lo adquieren, aunque puedan verse las consecuencias, a posteriori, cuando enfrentan otro conflicto. 
La mediación, y sus correlativas herramientas, son algunos de los aportes en los que consideramos que el Derecho se ha vuelto más "sensible" al abordaje de realidades sociales complejas. Dentro de esta perspectiva se presenta aquí a los Estudios sobre Discurso, como campo de investigación social, capaz de aportar herramientas teóricoconceptuales específicas para hacer frente a la comprensión de los procesos de interacción entre sujetos (individuales o colectivos). Por ello, es en esta dirección que avanzaremos en los apartados siguientes, intentando dar cuenta no solo de las posibilidades de esta línea de trabajo en tanto instrumento de intervención social sino como herramienta heurística sólida y confiable, que habiéndose desarrollado desde posicionamientos epistemológicos cualitativos se ha configurado como practica científica.

\subsection{LOS ESTUDIOS SOBRE DISCURSO EN EL MARCO DE LA INDAGACIÓN CIENTÍFICA CUALITATIVA.}

La metodología cualitativa, entendida como una corriente cada vez más vigorosa dentro del campo de las ciencias sociales, puede caracterizarse en primera instancia como un tipo de indagación que, mediante unidades no discretas, pretende describir y explicar determinados fenómenos de la realidad social.

Se trata de un modo de relevamiento que encuentra insuficiente el empleo de los números y las representaciones estadísticas para comprender ciertos procesos del comportamiento humano, individual o colectivo.

La emergencia de ciertos postulados filosóficos, provenientes de la fenomenología o el pragmatismo, dieron a finales del S. XIX lugar a que pudiera constituirse un paradigma epistemológico que cuestionara drásticamente el modelo positivista, que aún siendo originario de las ciencias denominadas 'naturales' había logrado por entonces constituirse como 'hegemónico' también en las prácticas científicas sociales. 
Santiago Castro Gómez (2007:79-91), como uno de los exponentes más relevantes de la corriente teórica crítica latinoamericana, ha denominado 'Hybris del punto cero' a la ilusión científica promovida desde la modernidad europea que creyó posible alcanzar un punto inobservado de observación. Algo análogo al concepto de panóptico, referido por Foucault, que culminaría en una descripción veraz de la realidad, logrando instituir un solo saber científico como universal.

El constructivismo como postura ontológica es el cimiento para erigir una serie de teorías que, desde allí, se proponen dilucidar de qué modo la construcción social de sentido condiciona el curso de ciertos acontecimientos humanos.

Esta corriente científica ya no estaría entonces abocada a des-cubrir una realidad externa a la subjetividad sino que, por el contrario, debería comprometerse a encontrar caminos metodológicos que permitieran acceder de un modo eficaz a estas elaboraciones abstractas, sutiles e imprecisas. El desafío consistiría, a partir de entonces, en alcanzar estas informaciones que, generando una infinita red de significados desde los sentidos, las emociones y la razón, operarían como dispositivos omnipresentes mediando nuestra relación con lo real.

De manera similar, la pretensión de 'generalización' es un punto de renuncia para los enfoques cualitativos que no aspiran, mediante el estudio de casos por ejemplo, elaborar 'leyes' aplicables a toda conducta social sino, que de modo muy diferente, están orientados a comprender singularidades.

El conocimiento resultante no podrá entonces ser usado como 'predicción' para anticipar tendencias en próximos procesos de determinadas características sino que, homomórficamente, podrá ser empleado como insumo para comprender otros casos, incluso pertenecientes a otros universos sociales. 
Por otra parte y como lo señala Parisí (2009:27) la noción misma de 'empírico' se cuestiona y enriquece desde estos enfoques; pues no se ciñe a lo observable, manipulable y medible, sino que permite pensar a la teoría como estrategia de construcción del objeto de estudio posibilitando el cruce entre teorías y objetos más complejos.

El fenómeno no pre-existe a la mirada del investigador ni a la subjetividad puesta en consideración; adquiere entidad en la medida en que estas dos posiciones generan instancias de diálogo y encuentro en las que el contorno del 'problema' va siendo definido por las producciones de sentido de las partes intervinientes.

Las corrientes hermenéuticas imbuidas por el inter-accionismo simbólico han encontrado en estas sendas científicas una multiplicidad de métodos en tanto recursos eficientes para explicar la relación, siempre dinámica, entre estructura y agencia social. La etnografía, la etnometodología, el estudio de casos, la reconstrucción biográfica, la investigación-acción o la teoría fundada son buenos ejemplos de abordajes metodológicos que, desde diferentes estrategias iluminan aquellos aspectos más escurridizos que logran explicar hoy a todo orden social como resultado de una serie de elementos originados en un sistema cultural.

Ahora bien, estas mismas consideraciones, descriptas aquí de modo sucinto, han servido para construir y deconstruir objetos de estudio; pero sus argumentos valen por supuesto de igual modo para explicar la 'naturaleza' de la investigación científica en tanto objeto. La idea de que toda representación mental, simple o compleja, es resultado de una serie de vinculaciones de sentido sirvió en primera instancia para comprender ciertos fenómenos del universo social, pero pronto se volvió sobre la propia acción investigativa. Es decir que un empleo cabal y maduro de este tipo de enfoque debe poner en consideración todos los puntos de anclaje cultural sobre los que se erige también la propia empresa científica. 
Reconocer las características del ámbito en el que se sitúa la actividad académica es un modo de ponerlo en consideración como campo, como espacio de pugnas atravesado por ciertas tensiones e intereses que constituyen el andamiaje ideológico y axiológico que sostiene el trabajo propuesto. En otras palabras, y desde el punto de vista de la aplicación, se trata de explicitar los valores que impulsan el planteamiento y la realización de una investigación dada, que jamás están restringidos a meros intereses gnoseológicos.

Cada paso en el diseño de una investigación, así como cada componente en la ejecución de cualquier acción, implica una selección de una entre diversas posibilidades (Rut Vieytes, 2009:43). El establecimiento de un tema de investigación implica, por ejemplo, un recorte sobre una determinada situación, privilegiando algunos aspectos del asunto en cuestión.

La metodología cualitativa, aún intentando salvar restricciones disciplinares que suelen coartar el desarrollo de cualquier investigación, asume que existe un punto de vista previo (ontológico, epistemológico y axiológico) que se pone en diálogo con el objeto de estudio elegido.

Del mismo modo, el empleo de un método de abordaje implica una elección sobre distintas opciones que, por supuesto, deberían estar en consonancia con el tipo de objeto construido desde la postulación del tema y la formulación de los objetivos directrices del proceso. Existen, por ello, numerosos modos de aproximación a un problema social dentro del amplio espectro de posibilidades que ofrece la metodología cualitativa.

Sin embargo, hay algo que podría denominarse 'común' a todo abordaje. Un elemento presente en las fases de construcción argumental, inicial y final, incluso de las indagaciones netamente cuantitativas. Se trata del empleo de mecanismos, más o menos 
concientes, de interpretación textual. Este proceso, que por extremadamente dinámico ha aparecido con frecuencia esquivo a la referencia teórica, ha sido muy estudiado por diversas corrientes dentro del área interdisciplinar de estudios semióticos.

El fundador de la tradición semiótica triádica, Charles Sanders Peirce (EP 2:411) ${ }^{4}$, aportó una noción nuclear que sería luego tomada para numerosos desarrollos teóricos producidos al interior de la sociología. Se trata del concepto de 'semiosis' que alude al modo en que los signos, significados y materialidades, se vinculan entre sí a través de una red infinita de sentido. Todo aquello que aparece ante nuestra percepción, dotado semánticamente, está vinculado a otros sentidos, y aquellos a su vez, a otras producciones anteriores ad infinitum.

Asociar en cada investigación el tema, los tipos y estrategias de abordaje a los contextos sociales y marcos culturales que les permitieron emergencia es imprescindible para comprender el modo en que el problema de investigación ha sido construido desde el momento inicial. Por otra parte, esta caracterización primera es necesaria para identificarla como elemento que dialogará con el sistema de representaciones de los sujetos involucrados como 'objeto' de indagación.

Las investigaciones netamente teóricas trabajan exclusivamente con insumos textuales que, mediante tareas de análisis conceptual, dan lugar a nuevas articulaciones de sentido generando nueva teoría social. Es sencillo identificar aquí el rol del científico con el del analista discursivo; pero en rigor toda investigación social, de nivel macro o micro, que tome fuentes primarias o secundarias, realiza siempre desde algún criterio, un trabajo de análisis de discurso. Las investigaciones que incluyen algún tipo de observación en campo o que emplean la entrevista como instrumento de indagación, también consisten estrictamente en trabajos de análisis. Toda la información generada, fuentes primarias en este último ejemplo, serán tomadas como fragmentos textuales

\footnotetext{
4 Se cita a Charles Sanders Peirce de modo convencional, en el caso de The Essential Peirce, las siglas EP van seguidas del número de volumen $\mathrm{y}$, tras los dos puntos (:), el número de página.
} 
relacionando los contenidos que en ellos residen con otros que resultan relevantes a los fines de la investigación planteada, sean éstos últimos elementos de la teoría o de contexto por ejemplo.

Del mismo modo, las investigaciones que trabajan con textos ya producidos en instancias previas, como es cualquier tipo de documentación, narrativa o estadística, terminan siempre por ordenar este material en el marco de sucesivas interpretaciones que articulan explicaciones sobre el fenómeno estudiado, generando nuevamente, teoría social.

Análisis del discurso es por ello, además de una línea metodológica, un modelo de indagación característico de todo proceso de investigación científica social.

Ahora bien, incluirlo como perspectiva de abordaje consiste precisamente, y como se mencionó antes, en hacer explícitas las relaciones de sentido que dieron lugar a la producción de un determinado texto y las efectivizadas en el momento de su lectura por el propio analista. Por lo tanto, y como tal, tampoco es una práctica exclusiva de las producciones científicas. Toda situación de comunicación, interpersonal desde una perspectiva microsociológica o interactoral en niveles macro y medios, incluye algún tipo de análisis discursivo ya que cada instancia de producción comporta siempre otra de lectura de otros fragmentos semánticos.

Desde la tradición francesa marxista, ubicando como punto relevante a Pecheux, hasta el modelo ternario recuperado por la semiótica de Eliseo Verón podemos encontrar una variedad de enfoques de los que aquí resultaría imposible dar cuenta. Sin embargo, podemos mencionar como punto recurrente y rasgo característico, la idea de que cada discurso, cada palabra proferida o escrita, dispone una serie de elementos que intentan definir, ante todo, un tipo de relación entre enunciador y enunciatario. 
Un discurso político, una conversación entre amigos, un artículo en un periódico o el texto de un libro son buenos ejemplos de fragmentos discursivos que, más allá de producir significado por la acepción de sus palabras, producen sentido en tanto refieren a otros discursos del contexto en que aparecen. Más allá de aquello que los diccionarios señalan sobre lo que las palabras indican, la producción del sentido viaja transformándose de acuerdo al contexto en el que tiene lugar.

La práctica jurídica entiende de ello, al reconocer que existen diversas interpretaciones orientadas por las diferentes posiciones e intereses de las partes enfrentadas que se proponen leer la letra de la propia ley ante una determinada situación de conflicto.

El análisis del discurso es una línea de estudio que no se propone, entonces, encontrar el significado "correcto" o "verdadero" de un conjunto de postulados sino que por el contrario, intenta evidenciar el modo en que esos textos se postulan como legítimos en diversas situaciones y para diversos actores.

Ahora bien, hasta aquí se ha hecho referencia al análisis del discurso, en tanto proceso de interpretación textual, como un componente ineludible en la investigación científica social; pero se ha mencionado asimismo que su aplicación no se restringe a la práctica científica. Cada instancia de interacción social, mediada discursivamente, constituye una situación de producción y lectura de múltiples y simultáneos planos discursivos.

Cada vez que una persona decide decir algo a otra, efectiviza toda una serie de mecanismos cognitivos que fundamentalmente están orientados a seleccionar información que resulta pertinente a ser proferida en la situación dada. Es decir que el locutor elegirá, de modo consciente e inconsciente, cada término empleado del amplio repertorio que la lengua le ofrece. Del mismo modo articulará estas palabras en singulares construcciones que habrá de elegir como convenientes, y por supuesto inteligibles. El criterio de selección para cada uno de estos momentos consistirá precisamente en un análisis de la situación a la que deberá encuadrar su propia 
expresión. Tendrá en cuenta las características de su locutor, se anticipará a lo que considerará son sus competencias comunicativas, y también en base a ello elegirá palabras y modos de ordenarlas para producir el sentido buscado.

Algo similar ocurre cuando la situación de comunicación se da a nivel medio o macro, teniendo por partes a diferentes actores sociales que como colectivos de sujetos también construyen sus discursos luego de analizar situaciones de enunciación, contextos y otros elementos.

Por tanto, y como podrá imaginarse ya, el conjunto de estrategias de análisis de discurso desarrollado por las ciencias sociales en general, y las metodologías cualitativas de corte semiótico en particular, ofrece elementos muy útiles a ser empleados en situaciones de interacción social que tienen como protagonistas a diversos grupos o sectores que, por determinadas razones, están involucrados en situaciones de conflicto. Por lo mismo, es imprescindible en estos casos contar con intervenciones calificadas que puedan atender estos aspectos condicionantes de las propias relaciones puestas en tensión.

En primer término, para iniciar un trabajo consciente de análisis, se deben identificar la o las tematizaciones que sostienen una determinada producción discursiva. Es decir que como punto de partida debe prestarse atención al modo en que cada actor, interviniente en una situación de conflicto, construye el tema de discrepancia que lo convoca. Es esperable que cada parte nomine de modo distinto la razón primera de un determinado problema, y que por tanto use una serie de categorías y conceptos alineados a su posición e intereses.

En el caso de disputas urbanas en torno al derecho de ocupación del suelo sucede con frecuencia que la misma situación es referida de modos, no sólo diversos sino en ciertas oportunidades, opuestos. 
Así por ejemplo, nominar aquellos asentamientos espontáneos e informales sobre terrenos fiscales o privados, como 'usurpaciones' comporta toda una posición en torno al problema, y señala fundamentalmente una perspectiva, un lugar ideológico y social, desde el cual se observa y entiende esta situación.

De modo similar, podemos reconocer que los discursos de las organizaciones sociales tendientes a apoyar a estas comunidades comprenden y expresan otro punto de vista que sin duda estará reflejado en el modo de referir a la situación de conflicto como 'amenaza de desalojos', 'violación de derechos humanos', etc.

El valor de emplear algunos elementos de análisis semiótico, orientados a comprender estos procesos y tensiones sociales, no reside en el propósito de encontrar la forma adecuada para referirlos sino en ser capaces de tender puentes de sentido entre unos y otros sectores.

Es decir que en un segundo paso deberían leerse, más detallada y atentamente, aquellas palabras o expresiones que aparecen como recurrentes o relevantes en cuanto a su peso en la construcción de un argumento.

Así como se explicaba más arriba que no es lo mismo titular a modo de síntesis un conflicto como 'usurpación del suelo' que como 'amenaza de desalojo', tampoco lo es el modo de nominar a los actores involucrados.

Resultaría obvio a primera vista decir que, en todas estas instancias de encuentro e inter-acción, los calificativos peyorativos son el obstáculo más frecuente o importante. Sin embargo, la astucia consiste en advertir los significados, que de modo más sutil se esconden tras ciertas expresiones a las que todos estamos, de un modo $u$ otro, habituados. Como señala Irene Vasilachis de Gialdino (2003: 103-116), respecto de la manera en que las personas que viven en la calle son representadas por ciertos medios de comunicación, emplear términos que definan por la negación a los sujetos referidos, 
es un modo de estigmatizar a estas personas. Este tipo de elección léxica constituye un obstáculo fundamental para sentar cualquier relación de paridad entre las propias personas involucradas, pensando por ejemplo, en quien usa estas expresiones, quien las escucha o lee, y quienes son efectivamente representados por ellas.

No se trata de advertir o condenar las malas intenciones de ciertas personas o grupos al utilizar determinado tipo de discurso; la propuesta consiste en comprender que todos, como sujetos vinculados a una serie de elementos culturales, estamos en relación a diversos marcos ideológicos que, como conjunto de proposiciones valorativas, orientan nuestra forma de percibir el mundo, los sujetos que en él se desempeñan y sus acciones.

Teun Van Dijk (2008) plantea que los diversos grupos ideológicos, que constituyen un cuerpo social, están fundamentalmente definidos por el modo en que construyen su identidad en relación a los sentimientos de pertenencia que el propio grupo logra suscitar por las tareas y actividades que cada sector desarrolla normalmente, por sus objetivos y propósitos, por sus normas y valores, por el acceso a los recursos -materiales o simbólicos-, pero también por la definición que cada grupo logra construir para sí, al diferenciarse de los otros.

Esta forma de comprender las producciones discursivas se considera aquí muy provechosa, ya que ayuda a pensar las tensiones entre diferentes actores sociales desde un enfoque relacional capaz de iluminar las razones profundas que provocan la emergencia de ciertos conflictos inter-sectoriales.

La disputa por el acceso a determinado bien material, como puede ser la tenencia o usufructo del suelo urbano, excede los intereses económicos de las partes enfrentadas. En todo caso, podría decirse que este aspecto, siendo un fuerte factor condicionante, está ligado a otra serie de elementos (identitarios, culturales e ideológicos) que también están operando fuertemente en estos procesos de interacción social. 
Tal como se señaló más arriba, toda mención a un suceso o hecho social implica disponer ciertos elementos en una estructura narrativa. Se trata de hacer, en primer término, una construcción de escenario, describiendo un espacio, físico o virtual, al que se caracteriza siempre de algún modo.

En el caso de los conflictos urbanos, un espacio recurrente descripto, es por supuesto la ciudad misma, siendo definida en sus características principales por la posición de quien la vive, entiende y por tanto, enuncia. Los sectores considerados medios y altos, en términos de clase, suelen referir a este espacio como un lugar que debe ser recuperado en términos de seguridad, orden y belleza. La amenaza a este proyecto desde luego está encarnada por los sectores sub-alternos que no sólo representan un peligro para el 'desarrollo armonioso' de la vida en los espacios públicos sino que asimismo constituye un elemento de 'deterioro estético'. Ya ha sido suficientemente señalado que la distancia entre distintos estratos sociales puede ser medida en relación a nivel de ingresos o grado de educación; pero aquellos aspectos relacionados a gustos y consumos resultan esquivos a la mesura numérica, aunque operan como factores igualmente condicionantes de las relaciones entre 'clases'.

La forma en que cada sector, constitutivo de un cuerpo social, ha aprendido a entender y valorar los diferentes aspectos éticos y estéticos de la vida en comunidad aparece en diversas oportunidades como posturas opuestas que generan fricciones ocasionando altercados de diversa intensidad y magnitud.

Es decir que la diferencia en el modo de comprender el escenario, en casos de disputas inter-actorales urbanas, constituye el primer elemento del conflicto mismo.

En segundo lugar aparece la caracterización de los sujetos (individuales o colectivos) que establecen el campo de vinculaciones. Esto es la definición relacional que cada grupo hace del resto, y que tal como se mencionaba antes, llega a manifestarse en términos de 'aliados' y 'adversarios'. En otras palabras, cada actor construye un espacio 
social virtual caracterizando posiciones y relaciones en función de sus propios intereses, definiendo al resto de los sujetos sociales como actores que facilitan u obstaculizan la concreción de los propios propósitos.

En un proceso de diálogo asistido, como puede considerarse la práctica de mediación, este componente respecto de las distintas apreciaciones del escenario, actores y conflictos resulta esencial, ya que será imprescindible orientar el proceso de comunicación inter-sectorial a cierta comunión interpretativa del problema. Asumiendo que cada actor conservará cierta especificidad en su modo de entender el problema y valorar los posibles caminos resolutivos, se intentará llegar a cierto consenso, al menos respecto de un desenlace que se perciba aceptable para todas la partes.

Escuchar, analizar, imaginar y ensayar nuevas formas de leer la situación, tomando elementos de los diferentes modos en que las partes narran el problema planteado, resulta indispensable.

El teórico británico Norman Fairclough (2003:75) , siendo uno de los representantes más reconocidos de la corriente de Análisis Crítico del Discurso, propone la noción de 'géneros' para caracterizar los diferentes modos de interactuar discursivamente.

Se trata de pensar las relaciones, inter-sectoriales por ejemplo, como concretizaciones de una serie de variables de sentido que determinan un tipo de relación entre las partes intervinientes. Siendo el discurso el medio, soporte y materialización de estas vinculaciones, es el óptimo objeto de consideración para describir y comprender los rasgos de estas relaciones.

De este modo, para atender a la paridad, jerarquía o distancia planteada entre las partes, será necesario revisar que todos los sectores involucrados tengan la misma posibilidad de verter sus opiniones o intereses, cuidando que el flujo de la información sea multi o bi-direccional; o dicho de otro modo, procurando que todos los actores 
involucrados estén en condiciones de proponer y replicar sentido, a través de sus intervenciones.

Vale aclarar aquí, que cualquiera de estos señalamientos se podría aplicar cada momento de las diferentes instancias que un proceso de mediación pueda constituir. Es decir, que estos ítems deben pensarse para todas las materializaciones discursivas posibles; ya sea para el manejo de la información escrita que aparezca en cierta documentación empleada o para la producción textual oral que pudiera suscitarse en un encuentro directo entre las partes. Cualquiera de estas situaciones se considerará fragmento discursivo del proceso, y deberá pensarse asimismo correlacionadamente.

\section{A MODO DE CONCLUSIÓN}

Reflexionar sobre las herramientas que podrían estrechar vínculos entre sectores urbanos movilizados por intereses diversos, y muchas veces contrapuestos, no es una tarea simple. Las Ciencias Sociales tienen deudas pendientes en la materia. La disciplina jurídica intenta saldar parte de este déficit al promover instituciones como la mediación.

Las nociones que propone la Teoría del Discurso aparecen al interior de esta práctica como instrumentos de intervención de status científico consolidado. Es necesario por ello, sacar provecho de los desarrollos hasta aquí realizados bajo el paradigma interpretativista que enmarca a todos aquellos abordajes cualitativos de los fenómenos sociales.

Sin embargo, somos conscientes de que las instituciones no son neutrales y por lo tanto están condicionadas por las circunstancias en las que se desenvuelven. Los contextos rígidos, competitivos y verticales, condicionan los diálogos y las herramientas de resolución de los conflictos. En este sentido, coincidimos con Schvarstein (2005: 28) al señalar que la mediación, como proceso social, se encuentra limitada por los acotados 
períodos de tiempo en que se desenvuelve, situación que dificulta la posibilidad de construir una relación alternativa.

Con esto no se pretende relativizar los aportes que este mismo trabajo busca realizar, sino por el contrario, se intenta aclarar que estas mismas reflexiones y las correlativas prácticas que el desarrollo de esta investigación ha tenido y tendrá, forman parte de órdenes y procesos más amplios que necesariamente deben ser puestos en consideración. Por tanto, las investigaciones sociales deberían poder dar cuenta, con aptitud explicativa, de las patologías estructurales de las sociedades contemporáneas, asumiendo asimismo el desafío de ampliar la escala de experiencias exitosas en pos de institucionalizar prácticas superadoras.

\section{BIBLIOGRAFÍA}

- ANDERSEN T.: “El equipo reflexivo" Barcelona, 1994. Editorial Gedisa.

- CARCOVA, C.: "Teorías jurídicas postpositivistas". Buenos Aires, 2009. Editorial Lexis Nexis.

- CASTRO GÓMEZ, S.: “Decolonizar la Universidad. La Hybris del punto cero y el diálogo de saberes". En Santiago Castro-Gómez y Ramón Grosfoguel (Eds.) El giro decolonial. (pp. 79-91) Bogotá, 2007. Siglo del Hombre Editores.

- FAIRClOUGH, N.: "Analysing Discourse: textual analysis for social research" Londres, 2003 London. Routledge.

- OST, F.: “Júpiter, Hércules y Hermes, tres modelos de juez" Alicante, 1992. en Doxa, n.12, pp. 170 ss. Universidad de Alicante.

- PARISÍ, A.: "Algunas reflexiones epistemológicas acerca de las ciencias sociales y la investigación cualitativa." En Aldo Merlino (Coord.) Investigación cualitativa en Ciencias Sociales. Temas problemas y aplicaciones. (pp.17-39). Buenos Aires, 2009. Cengage Learning. 
- PEIRCE, C.: "The Essential Peirce. Sellected Philosophical writings".Vol.2. 1893-1913. Bloomington, Indianapolis, 1998. Indiana University Press.

- SANTOS, B.: "Crítica de la razón indolente. Contra el desperdicio de la experiencia." Bilbao, 2003. Editorial Desclee de Brouwer.

- SANTOS, B.: La Reinvención solidaria y participativa del Estado en Boaventurade Sousa Santos "Reinventar la democracia. Reinventar el Estado". Buenos Aires, 2005. Clacso. Recuperado el 20 de enero de 2011. Acceso al texto completo : http:// bibliotecavirtual.clacso.org.ar/ar/libros/sousa/Reinventar\%20la\%20De mocracia.pdf

- SANTOS, B.: Para uma sociologia das ausencias e uma sociologia das emergencias. Revista Critica de Ciencias Sociais, Coimbra, 2002. 63, 237-280. Facultad de Economía de la Universidad de Coimbra.

- SARTORI, G.: "La sociedad multietnica. Pluralismo y extranjeros", Madrid,2001. Taurus.

- SCHVARSTEIN, L.: Prólogo a "Mediación, conducción de disputas, comunicación y técnicas". Buenos Aires, 2005. Paidos.

- SUARES M.: "Mediación, conducción de disputas, comunicación y técnicas."Buenos Aires, 2005. Editorial Paidos.

- VAN DIJK, T.: "Semántica del discurso e ideología". Discurso y Sociedad. Madrid, 2008. Vol. 2 (1), 201-261.

- VASILACHIS DE GIALDINO, I.: "Pobres, pobreza, identidad y representaciones sociales". Barcelona, 2003. Gedisa.

- VIEYTES, R.: Campos de aplicación y decisiones de diseño en la Investigación Cualitativa en En Aldo Merlino (Coord.) "Investigación cualitativa en Ciencias Sociales. Temas problemas y aplicaciones". (pp.43-84). Buenos Aires., 2009. Cengage Learning. 